\title{
Stability and $l_{2}$-gain analysis for 2D discrete switched systems with time-varying delays in the second FM model
}

\author{
Shipei Huang and Zhengrong Xiang*
}

\section{"Correspondence:}

xiangzr@mail.njust.edu.cn School of Automation, Nanjing University of Science and Technology, Nanjing, 210094, People's Republic of China

\begin{abstract}
This paper is concerned with the problems of stability and $I_{2}$-gain analysis for $2 \mathrm{D}$ (two-dimensional) discrete switched systems with time-varying delays described by the second FM state-space model. Firstly, we introduce the definition of the average dwell time for a 2D discrete switched system, which is an extension of the 'average dwell time' concept of a 1D (one-dimensional) switched system. Secondly, based on the average dwell time approach, delay-dependent sufficient conditions for the existence of the exponential stability for the 2D discrete switched system are derived and $/ 2$-gain performance for the considered system is also analyzed. All the obtained results are formulated in a set of LMIs (linear matrix inequalities). Finally, a numerical example is given to illustrate the effectiveness of the proposed results.
\end{abstract}

Keywords: 2D systems; switched systems; time-varying delays; /2-gain; average dwell time; linear matrix inequality

\section{Introduction}

2D (Two-dimensional) systems, which are a class of multi-dimensional systems, have received considerable attention over the past few decades due to their wide applications in many areas such as multi-dimensional digital filtering, linear image processing, signal processing, and process control [1-3]. The 2D system theory is frequently used as an analysis tool to solve some problems, e.g., iterative learning control $[4,5]$ and repetitive process control $[6,7]$. The problems on realization, stability analysis, stabilization, filter design, and so on for 2D or $n \mathrm{D}$ systems have attracted a great deal of interest by many researchers. $\mathrm{Xu}$ et al. [8,9] investigated the realization of 2D systems, and the problems of stability and stabilization for these systems were studied extensively in [10-17]. The observer and filter design problems have also been considered in [18-20].

It is known that modeling uncertainties and disturbances are unavoidable in practical systems, and it is important to investigate the problems of $H_{\infty}$ control and robust stabilization of 2D systems. Recently, many results on $H_{\infty}$ control for 2D systems have been presented in [21-23]. Because time delays frequently occur in practical systems and are often the source of instability, the $H_{\infty}$ control problem for a class of 2D discrete systems with state delays has also been investigated in $[24,25]$.

On the other hand, since switched systems have numerous applications in many fields, such as mechanical systems, automotive industry, switched power converters, this class of

\section{黛 Springer}

C) 2013 Huang and Xiang; licensee Springer. This is an Open Access article distributed under the terms of the Creative Commons Attribution License (http://creativecommons.org/licenses/by/2.0), which permits unrestricted use, distribution, and reproduction in any medium, provided the original work is properly cited. 
systems has also attracted considerable attention during the past several decades [26-33]. Recently, some approaches have been applied widely to deal with these systems; see, for example, [26-33] and references cited therein. As stated in [34,35], in many modeling problems of physical processes, a 2D switching representation is needed. One can cite a 2D physically based model for advanced power bipolar devices and heat flux switching and modulating in a thermal transistor. At present, there have been a few reports on 2D discrete switched systems. Benzaouia et al. [34] firstly considered 2D switched systems with arbitrary switching sequences, and the process of switch is considered as a Markovian jumping one. In addition, the stabilization problem of discrete 2D switched systems was also studied in [35]. In [36], we extended the concept of average dwell time in 1D switched systems to 2D switched systems and designed a switching rule to guarantee the exponential stability of 2D switched delay-free systems. However, to the best of our knowledge, no works have considered the disturbance attenuation property of 2D switched systems to date. Moreover, because of the complicated behavior caused by the interaction between the continuous dynamics and discrete switching, the problem of disturbance attenuation performance analysis for 2D switched systems is more difficult to study, and the methods proposed in [21-25] cannot be directly applied to such systems. This motivates the present study.

In this paper, we are interested in investigating the issues of the exponential stability and $l_{2}$-gain analysis for $2 \mathrm{D}$ discrete switched systems with time-varying delays represented by the second FM model. The main contributions of this paper can be summarized as follows: (i) Based on the average dwell time approach, a delay-dependent exponential stability criterion for such systems is obtained and formulated in terms of LMIs (linear matrix inequalities); (ii) The Lyapunov-Krasovskii function with exponential term, which is different from the previous ones, is constructed to investigate the stability of the considered systems; and (iii) In order to investigate the disturbance attenuation property of the considered systems, we for the first time introduce the concept of $l_{2}$-gain for a $2 \mathrm{D}$ switched system, which is an extension of the $l_{2}$-gain performance index in the $1 \mathrm{D}$ case. The $l_{2}$-gain performance index can characterize the disturbance attenuation property of the underlying systems, and then, based on the established stability results, delay-dependent sufficient conditions for the existence of $l_{2}$-gain performance are derived in terms of LMIs, which can be easily verified by using some standard numerical software. The proposed method can also be applied to non-switched 2D discrete linear systems.

This paper is organized as follows. In Section 2, problem formulation and some necessary lemmas are given. In Section 3, based on the average dwell time approach, delaydependent sufficient conditions for the existence of the exponential stability and $l_{2}$-gain property are derived in terms of a set of matrix inequalities. A numerical example is provided to illustrate the effectiveness of the proposed approach in Section 4. Concluding remarks are given in Section 5.

Notations Throughout this paper, the superscript ' $T$ ' denotes the transpose, and the notation $X \geq Y(X>Y)$ means that matrix $X-Y$ is positive semi-definite (positive definite, respectively). $\|\cdot\|$ denotes the Euclidean norm. $I$ represents an identity matrix with an appropriate dimension. $\operatorname{diag}\left\{a_{i}\right\}$ denotes a diagonal matrix with the diagonal elements $a_{i}$, $i=1,2, \ldots, n . X^{-1}$ denotes the inverse of $X$. The asterisk $*$ in a matrix is used to denote the term that is induced by symmetry. The set of all nonnegative integers is represented 
by $Z_{+}$. The $l_{2}$ norm of a $2 \mathrm{D}$ signal $w(i, j)$ is given by

$$
\|w\|_{2}=\sqrt{\sum_{i=0}^{\infty} \sum_{j=0}^{\infty}\|w(i, j)\|^{2}}
$$

and $w(i, j)$ belongs to $l_{2}\{[0, \infty),[0, \infty)\}$ if $\|w\|_{2}<\infty$

\section{Problem formulation and preliminaries}

Consider the following 2D discrete switched systems with time-varying delays described by the second FM model:

$$
\begin{aligned}
& x(i+1, j+1)= A_{1}^{\sigma(i, j+1)} x(i, j+1)+A_{2}^{\sigma(i+1, j)} x(i+1, j)+A_{d 1}^{\sigma(i, j+1)} x\left(i-d_{1}(i), j+1\right) \\
&+A_{d 2}^{\sigma(i+1, j)} x\left(i+1, j-d_{2}(j)\right)+B_{1}^{\sigma(i, j+1)} w(i, j+1)+B_{2}^{\sigma(i+1, j)} w(i+1, j), \\
& z(i, j)=H^{\sigma(i, j)} x(i, j)+L^{\sigma(i, j)} w(i, j)
\end{aligned}
$$

where $x(i, j) \in R^{n}$ is the state vector, $w(i, j) \in R^{q}$ is the noise input which belongs to $l_{2}\{[0, \infty),[0, \infty)\}, z(i, j) \in R^{p}$ is the controlled output. $i$ and $j$ are integers in $Z_{+} \cdot \sigma(i, j)$ : $Z_{+} \times Z_{+} \rightarrow \underline{N}=\{1,2, \ldots, N\}$ is the switching signal. $N$ is the number of subsystems. $\sigma(i, j)=k, k \in \underline{N}$, denotes that the $k$ th subsystem is active. $A_{1}^{k}, A_{2}^{k}, A_{d 1}^{k}, A_{d 2}^{k}, B_{1}^{k}, B_{2}^{k}, H^{k}$, and $L^{k}$ are constant matrices with appropriate dimensions. $d_{1}(i)$ and $d_{2}(j)$ are delays along horizontal and vertical directions, respectively. We assume that $d_{1}(i)$ and $d_{2}(j)$ satisfy

$$
\underline{d}_{1} \leq d_{1}(i) \leq \bar{d}_{1}, \quad \underline{d}_{2} \leq d_{2}(j) \leq \bar{d}_{2}
$$

where $\underline{d}_{1}, \bar{d}_{1}, \underline{d}_{2}$, and $\bar{d}_{2}$ denote the lower and upper delay bounds along horizontal and vertical directions, respectively.

In this paper, it is assumed that the switch occurs only at each sampling point of $i$ or $j$. The switching sequence can be described as

$$
\left(\left(i_{0}, j_{0}\right), \sigma\left(i_{0}, j_{0}\right)\right),\left(\left(i_{1}, j_{1}\right), \sigma\left(i_{1}, j_{1}\right)\right), \ldots,\left(\left(i_{\pi}, j_{\pi}\right), \sigma\left(i_{\pi}, j_{\pi}\right)\right), \ldots
$$

where $\left(i_{\pi}, j_{\pi}\right)$ denotes the $\pi$ th switching instant. It should be noted that the value of $\sigma(i, j)$ only depends upon $i+j$ (see the references $[35,36]$ ).

Remark 1 If there is only one subsystem in system (1), it will degenerate to the following 2D system:

$$
\begin{aligned}
& x(i+1, j+1)= A_{1} x(i, j+1)+A_{2} x(i+1, j)+A_{d 1} x\left(i-d_{1}(i), j+1\right) \\
&+A_{d 2} x\left(i+1, j-d_{2}(j)\right)+B_{1} w(i, j+1)+B_{2} w(i+1, j), \\
& z(i, j)=H x(i, j)+L w(i, j) .
\end{aligned}
$$

Therefore, the addressed system (1) can be viewed as an extension of 2D time-varying delays systems to switched systems. 
For system (1), we consider a finite set of initial conditions, that is, there exist positive integers $z_{1}$ and $z_{2}$ such that

$$
\left\{\begin{array}{l}
x(i, j)=h_{i j}, \quad \forall 0 \leq j \leq z_{2}, i=-\bar{d}_{1},-\bar{d}_{1}+1, \ldots, 0, \\
x(i, j)=v_{i j}, \quad \forall 0 \leq i \leq z_{1}, j=-\bar{d}_{2},-\bar{d}_{2}+1, \ldots, 0, \\
h_{00}=v_{00}, \\
x(i, j)=0, \quad \forall j>z_{2}, i=-\bar{d}_{1},-\bar{d}_{1}+1, \ldots, 0 \\
x(i, j)=0, \quad \forall i>z_{1}, j=-\bar{d}_{2},-\bar{d}_{2}+1, \ldots, 0
\end{array}\right.
$$

where $z_{1}<\infty$ and $z_{2}<\infty$ are positive integers, $h_{i j}$ and $v_{i j}$ are given vectors.

Definition 1 System (1) with $w(i, j)=0$ is said to be exponentially stable under the switching signal $\sigma(i, j)$ if for a given $Z \geq 0$, there exist positive constants $c$ and $\xi$ such that

$$
\sum_{i+j=D}\|x(i, j)\|^{2} \leq \xi e^{-c(D-Z)} \sum_{i+j=Z}\|x(i, j)\|_{C}^{2}
$$

holds for all $D \geq Z$, where

$$
\begin{aligned}
& \sum_{i+j=Z}\|x(i, j)\|_{C}^{2} \triangleq \sup _{\substack{-\bar{d}_{1} \leq \theta_{h} \leq 0,-\bar{d}_{2} \leq \theta_{\nu} \leq 0}} \sum_{i+j=Z}\left\{\left\|x\left(i-\theta_{h}, j\right)\right\|^{2},\left\|x\left(i, j-\theta_{\nu}\right)\right\|^{2},\right. \\
& \left.\left\|\eta\left(i-\theta_{h}, j\right)\right\|^{2},\left\|\delta\left(i, j-\theta_{v}\right)\right\|^{2}\right\}, \\
& \eta\left(i-\theta_{h}, j\right)=x\left(i-\theta_{h}+1, j\right)-x\left(i-\theta_{h}, j\right), \\
& \delta\left(i, j-\theta_{v}\right)=x\left(i, j-\theta_{v}+1\right)-x\left(i, j-\theta_{v}\right) .
\end{aligned}
$$

Remark 2 From Definition 1, it is easy to see that when $Z$ is given, $\sum_{i+j=Z}\|x(i, j)\|_{C}^{2}$ will be bounded and $\sum_{i+j=D}\|x(i, j)\|^{2}$ will tend to be zero exponentially as $D$ goes to infinity, which also means $\|x(i, j)\|$ will tend to be zero exponentially.

Definition 2 [36] For any $i+j=D>Z=i_{Z}+j_{Z}$, let $N_{\sigma(i, j)}(Z, D)$ denote the switching number of the switching signal $\sigma(i, j)$ on an interval $(Z, D)$. If

$$
N_{\sigma(i, j)}(Z, D) \leq N_{0}+\frac{D-Z}{\tau_{a}}
$$

holds for given $N_{0} \geq 0, \tau_{a} \geq 0$, then the constant $\tau_{a}$ is called the average dwell time and $N_{0}$ is the chatter bound.

Remark 3 Definition 2 is an extension of the 'average dwell time' concept in a 1D switched system, which can be seen in [26]. In what follows, based on the extended average dwell time concept, we will investigate the problems of stability and $l_{2}$-gain analysis for a $2 \mathrm{D}$ discrete switched system with time-varying delays. It should be noted that we have studied the problems of stability analysis and stabilization of delay-free $2 \mathrm{D}$ switched systems using the average dwell time approach in [36]. 
Remark 4 Similar to the 1D switched system case, Definition 2 means that if there exists a positive number $\tau_{a}$ such that the switching signal $\sigma(i, j)$ has the average dwell time property, the average time interval between consecutive switching is at least $\tau_{a}$. The average dwell time method is used to restrict the switching number of the switching signal during a time interval such that the stability or other performances of the system can be guaranteed.

Definition 3 Consider 2D discrete switched system (1). For a given scalar $\gamma>0$, system (1) is said to have $l_{2}$-gain $\gamma$ under the switching signal $\sigma(i, j)$ if it satisfies the following conditions:

(1) When $w(i, j)=0$, system (1) is asymptotically stable;

(2) Under the zero boundary condition, it holds that

$$
\sum_{i=0}^{\infty} \sum_{j=0}^{\infty}\|\bar{z}\|_{2}^{2}<\gamma^{2} \sum_{i=0}^{\infty} \sum_{j=0}^{\infty}\|\bar{w}\|_{2}^{2}, \quad \forall 0 \neq w \in l_{2}\{[0, \infty),[0, \infty)\},
$$

where

$$
\|\bar{w}\|_{2}^{2}=\sum_{i=0}^{\infty} \sum_{j=0}^{\infty}\left\|\left[\begin{array}{l}
w(i, j+1) \\
w(i+1, j)
\end{array}\right]\right\|^{2}, \quad\|\bar{z}\|_{2}^{2}=\sum_{i=0}^{\infty} \sum_{j=0}^{\infty}\left\|\left[\begin{array}{l}
z(i, j+1) \\
z(i+1, j)
\end{array}\right]\right\|^{2} .
$$

Remark 5 It is not difficult to see that Definition 3 is an extension of the $l_{2}$-gain performance index in the $1 \mathrm{D}$ case. $\gamma$ characterizes the disturbance attenuation performance. The smaller $\gamma$ is, the better performance is.

Definition 4 Consider 2D discrete switched system (1). For a given scalar $\gamma>0$, system (1) is said to have weighted $l_{2}$-gain $\gamma$ under the switching signal $\sigma(i, j)$ if it satisfies the following conditions:

(1) When $w(i, j)=0$, system (1) is asymptotically stable;

(2) Under the zero boundary condition, it holds that

$$
\sum_{i=0}^{\infty} \sum_{j=0}^{\infty} \alpha^{i+j}\|\bar{z}\|_{2}^{2}<\gamma^{2} \sum_{i=0}^{\infty} \sum_{j=0}^{\infty}\|\bar{w}\|_{2}^{2}, \quad \forall 0 \neq w \in l_{2}\{[0, \infty),[0, \infty)\} .
$$

Remark 6 Similar to the 1D switched system case, Definition 4 means that system (1) can also have disturbances attenuation properties when it satisfies conditions (1) and (2) in Definition 4.

Lemma 1 Consider $2 D$ discrete switched system (1). Suppose that there exist a series of $C^{1}$ functions $V_{k}: R^{n} \rightarrow R(k \in \underline{N})$ and two positive scalars $\lambda_{1}$ and $\lambda_{2}$ for which the following inequality holds:

$$
\lambda_{1}\|x(i, j)\|^{2} \leq V_{k}(x(i, j)) \leq \lambda_{2}\|x(i, j)\|_{C}^{2}, \quad \forall i, j \in Z_{+}, \forall k \in \underline{N}
$$

if there exists a number $0<\alpha<1$ for which $V_{k}(x(i, j))$ along with the solution of system (1) satisfies the inequality

$$
\sum_{i+j=D} V_{k}(x(i, j)) \leq \alpha \sum_{i+j=D-1} V_{k}(x(i, j)), \quad D>Z=\max \left(z_{1}, z_{2}\right), \quad \forall k \in \underline{N},
$$


and $\mu \geq 1$ such that

$$
\sum_{i+j=m_{\pi}} V_{\sigma\left(i_{\pi}, j_{\pi}\right)}(x(i, j)) \leq \mu \sum_{i+j=\left(m_{\pi}\right)^{-}} V_{\sigma\left(i_{\pi-1}, j_{\pi-1}\right)}(x(i, j)), \quad \pi=1,2, \ldots
$$

then $2 D$ discrete switched system (1) is exponentially stable for every switching signal with the average dwell time scheme

$$
\tau_{a}>\tau_{a}^{*}=\frac{\ln \mu}{-\ln \alpha} .
$$

Proof Let $\chi=N_{\sigma(i, j)}(Z, D)$ denote the switch number of switching $\sigma(i, j)$ on an interval $[Z, D)$, and let $\left(i_{\pi-\chi+1}, j_{\pi-\chi+1}\right),\left(i_{\pi-\chi+2}, j_{\pi-\chi+2}\right), \ldots,\left(i_{\pi}, j_{\pi}\right)$ denote the switching points of $\sigma(i, j)$ over the interval $[Z, D)$. Denoting $m_{i}=i_{i}+j_{i}, i=\pi-\chi+1, \ldots, \pi$, it follows from (8) and (9) that

$$
\begin{aligned}
\sum_{i+j=D} V_{\sigma\left(i_{\pi}, j_{\pi}\right)}(x(i, j)) & <\alpha^{D-m_{\pi}} \sum_{i+j=m_{\pi}} V_{\sigma\left(i_{\pi}, j_{\pi}\right)}(x(i, j)) \\
& \leq \mu \alpha^{D-m_{\pi}} \sum_{i+j=\left(m_{\pi}\right)^{-}} V_{\sigma\left(i_{\pi-1}, j_{\pi-1}\right)}(x(i, j)) \\
& <\mu^{2} \alpha^{D-m_{\pi}} \alpha^{m_{\pi}-m_{\pi-1}} \sum_{i+j=\left(m_{\pi-1}\right)^{-}} V_{\sigma\left(i_{\pi-2}, j_{\pi-2}\right)}(x(i, j)) \\
& =\mu^{2} \alpha^{D-m_{\pi-1}} \sum_{i+j=\left(m_{\pi-1}\right)^{-}} V_{\sigma\left(i_{\pi-2}, j_{\pi-2}\right)}(x(i, j))<\cdots \\
& <\mu^{\chi} \alpha^{D-Z} \sum_{i+j=Z} V_{\sigma\left(i_{\pi-\chi}, j_{\pi-\chi}\right)}(x(i, j)) .
\end{aligned}
$$

According to Definition 2, one obtains

$$
\chi=N_{\sigma(i, j)}(Z, D) \leq N_{0}+\frac{D-Z}{\tau_{a}} .
$$

Then from (12), we have

$$
\begin{aligned}
& \sum_{i+j=D} V_{\sigma\left(i_{\pi}, j_{\pi}\right)}(x(i, j)) \\
& <\mu^{\chi} \alpha^{D-Z} \sum_{i+j=Z} V_{\sigma\left(i_{\pi-\chi}, j_{\pi-\chi}\right)}(x(i, j)) \\
& \quad=\mu^{N_{0}} e^{-\left(-\frac{\ln \mu}{\tau_{a}}-\ln \alpha\right)(D-Z)} \sum_{i+j=Z} V_{\sigma\left(i_{\pi-\chi}, j_{\pi-\chi}\right)}(x(i, j)) .
\end{aligned}
$$

From (7), we get

$$
\sum_{i+j=D}\|x(i, j)\|^{2} \leq \lambda_{1}^{-1} \lambda_{2} \mu^{N_{0}} e^{-\left(-\frac{\ln \mu}{\tau_{a}}-\ln \alpha\right)(D-Z)} \sum_{i+j=Z}\|x(i, j)\|_{C}^{2}
$$

Therefore, according to Definition 1, system (1) is exponentially stable under the average dwell time scheme (10). 


\section{Main results}

\subsection{Stability analysis}

Theorem 1 Consider system (1) with $w(i, j)=0$. For a given positive constant $\alpha<1$, if there exist positive definite symmetric matrices $P_{h}^{k}, P_{v}^{k}, Q_{h}^{k}, Q_{v}^{k}, W_{h}^{k}, W_{v}^{k}, R_{h}^{k}, R_{v}^{k}$, and matrices $N_{1}^{k}=\left[\begin{array}{c}N_{11}^{k} \\ N_{12}^{k}\end{array}\right], N_{2}^{k}=\left[\begin{array}{c}N_{21}^{k} \\ N_{22}^{k}\end{array}\right], M_{1}^{k}=\left[\begin{array}{c}M_{11}^{k} \\ M_{12}^{k}\end{array}\right], M_{2}^{k}=\left[\begin{array}{c}M_{21}^{k} \\ M_{22}^{k}\end{array}\right], X^{k}=\left[\begin{array}{cc}X_{11}^{k} & X_{12}^{k} \\ * & X_{22}^{k}\end{array}\right]>0$ and $Y^{k}=\left[\begin{array}{cc}Y_{11}^{k} & Y_{12}^{k} \\ * & Y_{22}^{k}\end{array}\right]>0$ with appropriate dimensions, $k \in \underline{N}$, such that

$$
\begin{aligned}
& {\left[\begin{array}{cccc}
\Gamma & \Xi_{1}^{T}\left(P_{h}^{k}+P_{v}^{k}\right) & \sqrt{\bar{d}_{1}} \Xi_{2}^{T} R_{h}^{k} & \sqrt{\bar{d}_{2}} \Xi_{3}^{T} R_{v}^{k} \\
* & -\left(P_{h}^{k}+P_{v}^{k}\right) & 0 & 0 \\
* & * & -R_{h}^{k} & 0 \\
* & * & * & -R_{v}^{k}
\end{array}\right]<0,} \\
& {\left[\begin{array}{cc}
X^{k} & N_{1}^{k} \\
* & R_{h}^{k}
\end{array}\right] \geq 0, \quad\left[\begin{array}{cc}
X^{k} & N_{2}^{k} \\
* & R_{h}^{k}
\end{array}\right] \geq 0,} \\
& {\left[\begin{array}{cc}
Y^{k} & M_{1}^{k} \\
* & R_{v}^{k}
\end{array}\right] \geq 0, \quad\left[\begin{array}{cc}
Y^{k} & M_{2}^{k} \\
* & R_{v}^{k}
\end{array}\right] \geq 0 \text {, }}
\end{aligned}
$$

where

$$
\begin{aligned}
& \Xi_{1}=\left[\begin{array}{llllll}
A_{1}^{k} & A_{2}^{k} & A_{d 1}^{k} & A_{d 2}^{k} & 0 & 0
\end{array}\right], \\
& \Xi_{2}=\left[\begin{array}{llllll}
A_{1}^{k}-I & A_{2}^{k} & A_{d 1}^{k} & A_{d 2}^{k} & 0 & 0
\end{array}\right], \\
& \Xi_{3}=\left[\begin{array}{llllll}
A_{1}^{k} & A_{2}^{k}-I & A_{d 1}^{k} & A_{d 2}^{k} & 0 & 0
\end{array}\right], \\
& \Gamma=\left[\begin{array}{cccccc}
\Gamma_{11} & 0 & \Gamma_{13} & 0 & -\alpha^{\bar{d}_{1}} N_{21}^{k} & 0 \\
* & \Gamma_{22} & 0 & \Gamma_{24} & 0 & -\alpha^{\bar{d}_{2}} M_{21}^{k} \\
* & * & \Gamma_{33} & 0 & -\alpha^{\bar{d}_{1}} N_{22}^{k} & 0 \\
* & * & * & \Gamma_{44} & 0 & -\alpha^{\bar{d}_{2}} M_{22}^{k} \\
* & * & * & * & -\alpha^{\bar{d}_{1}} W_{h}^{k} & 0 \\
* & * & * & * & * & -\alpha^{\bar{d}_{2}} W_{v}^{k}
\end{array}\right], \\
& \Gamma_{11}=-\alpha P_{h}^{k}+W_{h}^{k}+\left(\bar{d}_{1}-\underline{d}_{1}+1\right) Q_{h}^{k}+\alpha^{\bar{d}_{1}}\left(N_{11}^{k}+N_{11}^{k T}\right)+\bar{d}_{1} \alpha^{\bar{d}_{1}} X_{11}^{k}, \\
& \Gamma_{22}=-\alpha P_{v}^{k}+W_{v}^{k}+\left(\bar{d}_{2}-\underline{d}_{2}+1\right) Q_{v}^{k}+\alpha^{\bar{d}_{2}}\left(M_{11}^{k}+M_{11}^{k T}\right)+\bar{d}_{2} \alpha^{\bar{d}_{2}} Y_{11}^{k}, \\
& \Gamma_{13}=-\alpha^{\bar{d}_{1}}\left(N_{11}^{k}-N_{21}^{k}-N_{12}^{k T}\right)+\bar{d}_{1} \alpha^{\bar{d}_{1}} X_{12}^{k}, \\
& \Gamma_{24}=-\alpha^{\bar{d}_{2}}\left(M_{11}^{k}-M_{21}^{k}-M_{12}^{k T}\right)+\bar{d}_{2} \alpha^{\bar{d}_{2}} Y_{12}^{k}, \\
& \Gamma_{33}=-\alpha^{\bar{d}_{1}} Q_{h}^{k}+\alpha^{\bar{d}_{1}}\left(N_{22}^{k}+N_{22}^{k T}-N_{12}^{k}-N_{12}^{k T}\right)+\bar{d}_{1} \alpha^{\bar{d}_{1}} X_{22}^{k}, \\
& \Gamma_{44}=-\alpha^{\bar{d}_{2}} Q_{v}^{k}+\alpha^{\bar{d}_{2}}\left(M_{22}^{k}+M_{22}^{k T}-M_{12}^{k}-M_{12}^{k T}\right)+\bar{d}_{2} \alpha^{\bar{d}_{2}} Y_{22}^{k},
\end{aligned}
$$

hold, then under the average dwell time scheme

$$
\tau_{a}>\tau_{a}^{*}=\frac{\ln \mu}{-\ln \alpha}
$$


where $\mu \geq 1$ and satisfies

$$
\begin{array}{lcc}
P_{h}^{k} \leq \mu P_{h}^{l}, & P_{h}^{l} \leq \mu P_{h}^{k}, & Q_{h}^{k} \leq \mu Q_{h}^{l}, \\
Q_{h}^{l} \leq \mu Q_{h}^{k}, & W_{h}^{k} \leq \mu W_{h}^{l}, & \\
W_{h}^{l} \leq \mu W_{h}^{k}, & R_{h}^{k} \leq \mu R_{h}^{l}, & R_{h}^{l} \leq \mu R_{h}^{k}, \\
P_{v}^{k} \leq \mu P_{v}^{l}, & P_{v}^{l} \leq \mu P_{v}^{k}, \quad Q_{v}^{k} \leq \mu Q_{v}^{l}, \\
Q_{v}^{l} \leq \mu Q_{v}^{k}, & W_{v}^{k} \leq \mu W_{v}^{l}, \quad W_{v}^{l} \leq \mu W_{v}^{k}, \\
R_{v}^{k} \leq \mu R_{v}^{l}, & R_{v}^{l} \leq \mu R_{v}^{k}, \quad \forall k, l \in \underline{N},
\end{array}
$$

the system is exponentially stable.

Proof See the Appendix for the detailed proof, it is omitted here.

Remark 7 In Theorem 1, we propose a sufficient condition for the existence of exponential stability for the considered 2D discrete switched system (1). It is worth noting that this condition is obtained by using the average dwell time approach.

\section{2 $I_{2}$-gain performance analysis}

Theorem 2 Consider system (1). For given positive constants $\gamma$ and $\alpha<1$, if there exist positive definite symmetric matrices $P_{h}^{k}, P_{v}^{k}, Q_{h}^{k}, Q_{v}^{k}, W_{h}^{k}, W_{v}^{k}, R_{h}^{k}, R_{v}^{k}$, and matrices $N_{1}^{k}=$ $\left[\begin{array}{c}N_{11}^{k} \\ N_{12}^{k}\end{array}\right], N_{2}^{k}=\left[\begin{array}{c}N_{21}^{k} \\ N_{22}^{k}\end{array}\right], M_{1}^{k}=\left[\begin{array}{c}M_{11}^{k} \\ M_{12}^{k}\end{array}\right], M_{2}^{k}=\left[\begin{array}{c}M_{21}^{k} \\ M_{22}^{k}\end{array}\right], X^{k}=\left[\begin{array}{cc}X_{11}^{k} & X_{12}^{k} \\ * & X_{22}^{k}\end{array}\right]>0$ and $Y^{k}=\left[\begin{array}{cc}Y_{11}^{k} & Y_{12}^{k} \\ * & Y_{22}^{k}\end{array}\right]>0$ with appropriate dimensions, $k \in \underline{N}$, such that (16) and the following inequality

$$
\left[\begin{array}{ccccccc}
\Gamma & 0 & 0 & \Xi_{1}^{T}\left(P_{h}^{k}+P_{v}^{k}\right) & \sqrt{\bar{d}_{1}} \Xi_{2}^{T} R_{h}^{k} & \sqrt{\bar{d}_{2}} \Xi_{3}^{T} R_{v}^{k} & \Xi_{4}^{T} \\
* & -\gamma^{2} I & 0 & B_{1}^{T}\left(P_{h}^{k}+P_{v}^{k}\right) & \sqrt{\bar{d}_{1}} B_{1}^{T} R_{h}^{k} & \sqrt{\bar{d}_{2}} B_{1}^{T} R_{v}^{k} & L_{k}^{T} \\
* & * & -\gamma^{2} I & B_{2}^{T}\left(P_{h}^{k}+P_{v}^{k}\right) & \sqrt{\bar{d}_{1}} B_{2}^{T} R_{h}^{k} & \sqrt{\bar{d}_{2}} B_{2}^{T} R_{v}^{k} & L_{k}^{T} \\
* & * & * & -\left(P_{h}^{k}+P_{v}^{k}\right) & 0 & 0 & 0 \\
* & * & * & * & -R_{h}^{k} & 0 & 0 \\
* & * & * & * & * & -R_{v}^{k} & 0 \\
* & * & * & * & * & * & -I
\end{array}\right]<0,
$$

where

$$
\Xi_{4}=\left[\begin{array}{llllll}
H^{k} & H^{k} & 0 & 0 & 0 & 0
\end{array}\right]
$$

hold, then under the average dwell time scheme (17), the system is exponentially stable and has weighted $l_{2}$-gain $\gamma$.

Proof It is easy to get that (15) can be deduced from (19), and according to Theorem 1, we can obtain that system (1) is exponentially stable.

Now we are in a position to consider the $l_{2}$-gain performance of system (1) under the zero boundary condition. Following the proof line of Theorem 1, we get the following 
relationship for the $k$ th subsystem:

$$
\begin{aligned}
& V_{k}^{h}(i+1, j+1)+V_{k}^{v}(i+1, j+1) \\
& -\alpha V_{k}^{h}(i, j+1)-\alpha V_{k}^{v}(i+1, j)+\|z\|_{2}^{2}-\gamma^{2}\|w\|_{2}^{2} \\
& \leq\left[\begin{array}{c}
x(i, j+1) \\
x(i+1, j) \\
x\left(i-d_{1}(i), j+1\right) \\
x\left(i+1, j-d_{2}(j)\right) \\
x\left(i-\bar{d}_{1}, j+1\right) \\
x\left(i+1, j-\bar{d}_{2}\right) \\
w(i, j+1) \\
w(i+1, j)
\end{array}\right]^{T}\left(\tilde{\Psi}+\left[\begin{array}{c}
A_{1}^{k T} \\
A_{2}^{k T} \\
A_{d 1}^{k T} \\
A_{d 2}^{k T} \\
0 \\
0 \\
B_{1}^{k T} \\
B_{2}^{k T}
\end{array}\right]\left(P_{h}^{k}+P_{v}^{k}\right)\left[\begin{array}{c}
A_{1}^{k T} \\
A_{2}^{k T} \\
A_{d 1}^{k T} \\
A_{d 2}^{k T} \\
0 \\
0 \\
B_{1}^{k T} \\
B_{2}^{k T}
\end{array}\right]\right. \\
& \left.+\left[\begin{array}{cc}
A_{1}^{k T}-I & A_{1}^{k T} \\
A_{2}^{k T} & A_{2}^{k T}-I \\
A_{d 1}^{k T} & A_{d 1}^{k T} \\
A_{d 2}^{k T} & A_{d 2}^{k T} \\
0 & 0 \\
0 & 0 \\
B_{1}^{k T} & B_{1}^{k T} \\
B_{2}^{k T} & B_{2}^{k T}
\end{array}\right]\left[\begin{array}{cc}
\bar{d}_{1} R_{h}^{k} & 0 \\
0 & \bar{d}_{2} R_{v}^{k}
\end{array}\right]\left[\begin{array}{cc}
A_{1}^{k T}-I & A_{1}^{k T} \\
A_{2}^{k T} & A_{2}^{k T}-I \\
A_{d 1}^{k T} & A_{d 1}^{k T} \\
A_{d 2}^{k T} & A_{d 2}^{k T} \\
0 & 0 \\
0 & 0 \\
B_{1}^{k T} & B_{1}^{k T} \\
B_{2}^{k T} & B_{2}^{k T}
\end{array}\right]\right) \\
& \times\left[\begin{array}{c}
x(i, j+1) \\
x(i+1, j) \\
x\left(i-d_{1}(i), j+1\right) \\
x\left(i+1, j-d_{2}(j)\right) \\
x\left(i-\bar{d}_{1}, j+1\right) \\
x\left(i+1, j-\bar{d}_{2}\right) \\
w(i, j+1) \\
w(i+1, j)
\end{array}\right] \\
& -\sum_{r=i-d_{1}(i)}^{i-1}\left[\begin{array}{c}
x(i, j+1) \\
\left(i-d_{1}(i), j+1\right) \\
\eta(r, j+1)
\end{array}\right]^{T}\left[\begin{array}{cc}
X^{k} & N_{1}^{k} \\
* & R_{h}^{k}
\end{array}\right]\left[\begin{array}{c}
x(i, j+1) \\
\left(i-d_{1}(i), j+1\right) \\
\eta(r, j+1)
\end{array}\right] \alpha^{\bar{d}_{1}} \\
& -\sum_{r=i-\bar{d}_{1}}^{i-d_{1}(i)-1}\left[\begin{array}{c}
x(i, j+1) \\
x\left(i-d_{1}(i), j+1\right) \\
\eta(r, j+1)
\end{array}\right]^{T}\left[\begin{array}{cc}
X^{k} & N_{2}^{k} \\
* & R_{h}^{k}
\end{array}\right]\left[\begin{array}{c}
x(i, j+1) \\
x\left(i-d_{1}(i), j+1\right) \\
\eta(r, j+1)
\end{array}\right] \alpha^{\bar{d}_{1}} \\
& -\sum_{t=j-d_{2}(j)}^{j-1}\left[\begin{array}{c}
x(i+1, j) \\
x\left(i+1, j-d_{2}(j)\right) \\
\delta(i+1, t)
\end{array}\right]^{T}\left[\begin{array}{cc}
Y^{k} & M_{1}^{k} \\
* & R_{v}^{k}
\end{array}\right]\left[\begin{array}{c}
x(i+1, j) \\
x\left(i+1, j-d_{2}(j)\right) \\
\delta(i+1, t)
\end{array}\right] \alpha^{\bar{d}_{2}} \\
& -\sum_{t=j-\bar{d}_{2}}^{j-d_{2}(j)-1}\left[\begin{array}{c}
x(i+1, j) \\
x\left(i+1, j-d_{2}(j)\right) \\
\delta(i+1, t)
\end{array}\right]^{T}\left[\begin{array}{cc}
Y^{k} & M_{2}^{k} \\
* & R_{v}^{k}
\end{array}\right]\left[\begin{array}{c}
x(i+1, j) \\
x\left(i+1, j-d_{2}(j)\right) \\
\delta(i+1, t)
\end{array}\right] \alpha^{\bar{d}_{2}},
\end{aligned}
$$


where

$$
\begin{aligned}
& \tilde{\Psi}=\left[\begin{array}{cccccccc}
\tilde{\Psi}_{11} & H^{k T} H^{k} & 0 & 0 & 0 & 0 & H^{k T} L^{k} & H^{k T} L^{k} \\
* & \tilde{\Psi}_{22} & 0 & 0 & 0 & 0 & H^{k T} L^{k} & H^{k T} L^{k} \\
* & * & -\alpha^{\bar{d}_{1}} Q_{h}^{k} & 0 & 0 & 0 & 0 & 0 \\
* & * & * & -\alpha^{\bar{d}_{2}} Q_{v}^{k} & 0 & 0 & 0 & 0 \\
* & * & * & * & -\alpha^{\bar{d}_{1}} W_{h}^{k} & 0 & 0 & 0 \\
* & * & * & * & * & -\alpha^{\bar{d}_{2}} W_{v}^{k} & 0 & 0 \\
* & * & * & * & * & * & \tilde{\Psi}_{77} & L^{k T} L^{k} \\
* & * & * & * & * & * & * & \tilde{\Psi}_{88}
\end{array}\right], \\
& \tilde{\Psi}_{11}=-\alpha P_{h}^{k}+W_{h}^{k}+\left(\bar{d}_{1}-\underline{d}_{1}+1\right) Q_{h}^{k}+H^{k T} H^{k}, \\
& \tilde{\Psi}_{22}=-\alpha P_{v}^{k}+W_{v}^{k}+\left(\bar{d}_{2}-\underline{d}_{2}+1\right) Q_{v}^{k}+H^{k T} H^{k}, \\
& \tilde{\Psi}_{77}=\tilde{\Psi}_{88}=L^{k T} L^{k}-\gamma^{2} I .
\end{aligned}
$$

Then by the Schur complement lemma, we can obtain from (16) and (19) that

$$
\begin{aligned}
& V_{k}^{h}(i+1, j+1)+V_{k}^{v}(i+1, j+1) \\
& \quad-\alpha V_{k}^{h}(i, j+1)-\alpha V_{k}^{v}(i+1, j)+\|z\|_{2}^{2}-\gamma^{2}\|w\|_{2}^{2}<0 .
\end{aligned}
$$

Summing up both sides of (20) from $D-2$ to 0 with respect to $j$ and 0 to $D-2$ with respect to $i$ and applying the zero boundary condition, one gets

$$
\begin{aligned}
\sum_{i+j=D} V_{\sigma\left(i_{\pi}, i_{\pi}\right)}(i, j)< & \alpha \sum_{i+j=D-1} V_{\sigma\left(i_{\pi}, i_{\pi}\right)}(i, j)-\sum_{i+j=D-2} \Gamma(i, j) \\
< & \alpha^{D-m_{\pi}} \sum_{i+j=m_{\pi}} V_{\sigma\left(i_{\pi}, i_{\pi}\right)}(i, j)-\sum_{m=m_{\pi}-1}^{D-2} \sum_{i+j=m} \alpha^{D-2-i-j} \Gamma(i, j) \\
\leq & \mu \alpha^{D-m_{\pi}} \sum_{i+j=\left(m_{\pi}\right)^{-}} V_{\sigma\left(i_{\pi-1}, i_{\pi-1}\right)}(i, j)-\sum_{m=m_{\pi}-1}^{D-2} \sum_{i+j=m} \alpha^{D-2-i-j} \Gamma(i, j) \\
< & \sum_{i+j=m_{\pi}-1} \mu \alpha^{D-\left(m_{\pi}-1\right)} V_{\sigma\left(i_{\pi-1}, i_{\pi-1}\right)}(i, j)-\mu \alpha^{D-m_{\pi}} \sum_{i+j=m_{\pi}-2} \Gamma(i, j) \\
& -\sum_{m=m_{\pi}-1}^{D-2} \sum_{i+j=m} \alpha^{D-2-i-j} \Gamma(i, j) \\
= & \sum_{i+j=m_{\pi}-1} \mu^{N_{\sigma(i, j)}(i+j, D)} \alpha^{D-\left(m_{\pi}-1\right)} V_{\sigma\left(i_{\pi-1}, i_{\pi-1}\right)}(i, j) \\
& -\sum_{m=m_{\pi}-2}^{D-2} \sum_{i_{i+j=m}} \mu^{N_{\sigma(i, j)}(i+j+1, D)} \alpha^{D-2-i-j} \Gamma(i, j) \\
< & \sum_{i+j=m_{\pi-1}} \mu^{N_{\sigma(i, j)}(i+j, D)} \alpha^{D-m_{\pi-1}} V_{\sigma\left(i_{\pi-1}, i_{\pi-1}\right)}(i, j) \\
& -\sum_{m=m_{\pi-1}-1}^{D-2} \sum_{i+j=m} \mu^{N_{\sigma(i, j)}(i+j+1, D)} \alpha^{D-2-i-j} \Gamma(i, j)
\end{aligned}
$$




$$
\begin{aligned}
& \leq \sum_{i+j=\left(m_{\pi-1}\right)^{-}} \mu^{N_{\sigma(i, j)}(i+j-1, D)} \alpha^{D-m_{\pi-1}} V_{\sigma\left(i_{\pi-2}, i_{\pi-2}\right)}(i, j) \\
& \quad-\sum_{m=m_{\pi-1}-1} \sum_{i+j=m} \mu^{N_{\sigma(i, j)}(i+j+1, D)} \alpha^{D-2-i-j} \Gamma(i, j) \\
& <\cdots \\
& <\sum_{i+j=1} \mu^{N_{\sigma(i, j)}(i+j, D)} \alpha^{D-1} V_{\sigma(0,1)}(i, j) \\
& \quad-\sum_{m=0}^{D-2} \sum_{i+j=m} \mu^{N_{\sigma(i, j)}(i+j+1, D)} \alpha^{D-2-i-j} \Gamma(i, j)
\end{aligned}
$$

where

$$
\Gamma(i, j)=\|\bar{z}\|_{2}^{2}-\gamma^{2}\|\bar{w}\|_{2}^{2}=\left\|\begin{array}{l}
z(i+1, j) \\
z(i, j+1)
\end{array}\right\|_{2}^{2}-\gamma^{2}\left\|\begin{array}{l}
w(i+1, j) \\
w(i, j+1)
\end{array}\right\|_{2}^{2}
$$

Under the zero initial condition, it holds that

$$
\sum_{i+j=1} \mu^{N_{\sigma(i, j)}(i+j, D)} \alpha^{D-1} V_{\sigma(0,1)}(i, j)=0
$$

Thus we have

$$
\sum_{m=0}^{D-2} \sum_{i+j=m} \mu^{N_{\sigma(i, j)}(i+j+1, D)} \alpha^{D-2-i-j} \Gamma(i, j)<-\sum_{i+j=D} V_{\sigma\left(i_{\pi}, i_{\pi}\right)}(i, j)<0 .
$$

Multiplying the both sides of (23) by $\mu^{-N_{\sigma(i, j)}(1, D)}$, we get the following inequality:

$$
\sum_{m=0}^{D-2} \sum_{i+j=m} \mu^{-N_{\sigma(i, j)}(1, i+j+1)} \alpha^{D-2-i-j} \Gamma(i, j)<0
$$

That is,

$$
\sum_{m=0}^{D-2} \sum_{i+j=m} \mu^{-N_{\sigma(i, j)}(1, i+j+1)} \alpha^{D-2-i-j}\|\bar{z}\|_{2}^{2}<\sum_{m=0}^{D-2} \sum_{i+j=m} \mu^{-N_{\sigma(i, j)}(1, i+j+1)} \alpha^{D-2-i-j}\|\bar{w}\|_{2}^{2} .
$$

Note that $N_{\sigma(i, j)}(1, i+j+1) \leq(i+j) / \tau_{a}$, then using (17), we have

$$
\mu^{-N_{\sigma(i, j)}(1, i+j+1)}=e^{-N_{\sigma(i, j)}(1, i+j+1) \ln \mu} \geq e^{(i+j) \ln \alpha} .
$$

It follows that

$$
\begin{gathered}
\sum_{m=0}^{D-2} \sum_{i+j=m} e^{(i+j) \ln \alpha} \alpha^{D-2-i-j}\|\bar{z}\|_{2}^{2}<\sum_{m=0}^{D-2} \sum_{i+j=m} \mu^{-N_{\sigma(i, j)}(1, i+j+1)} \alpha^{D-2-i-j}\|\bar{w}\|_{2}^{2} \\
\Rightarrow \sum_{m=0}^{D-2} \sum_{i+j=m} \alpha^{D-2}\|\bar{z}\|_{2}^{2}<\gamma^{2} \sum_{m=0}^{D-2} \sum_{i+j=m} \alpha^{D-2-i-j}\|\bar{w}\|_{2}^{2}
\end{gathered}
$$




$$
\begin{aligned}
& \Rightarrow \quad \sum_{D=2}^{\infty} \sum_{m=0}^{D-2} \sum_{i+j=m} \alpha^{D-2}\|\bar{z}\|_{2}^{2}<\gamma^{2} \sum_{D=2}^{\infty} \sum_{m=0}^{D-2} \sum_{i+j=m} \alpha^{D-2-i-j}\|\bar{w}\|_{2}^{2} \\
& \Rightarrow \quad \sum_{m=0}^{\infty} \sum_{i+j=m} \alpha^{i+j}\|\bar{z}\|_{2}^{2} \sum_{D=2+m}^{\infty} \alpha^{D-2-m}<\gamma^{2} \sum_{m=0}^{\infty} \sum_{i+j=m}\|\bar{w}\|_{2}^{2} \sum_{D=2+m}^{\infty} \alpha^{D-2-m} \\
& \Rightarrow \quad \frac{1}{1-\alpha} \sum_{m=0}^{\infty} \sum_{i+j=m} \alpha^{i+j}\|\bar{z}\|_{2}^{2}<\gamma^{2} \frac{1}{1-\alpha} \sum_{m=0}^{\infty} \sum_{i+j=m}\|\bar{w}\|_{2}^{2} \\
& \Rightarrow \quad \sum_{m=0}^{\infty} \sum_{i+j=m} \alpha^{i+j}\|\bar{z}\|_{2}^{2}<\gamma^{2} \sum_{m=0}^{\infty} \sum_{i+j=m}\|\bar{w}\|_{2}^{2} \\
& \Rightarrow \quad \sum_{i=0}^{\infty} \sum_{j=0}^{\infty} \alpha^{i+j}\|\bar{z}\|_{2}^{2}<\gamma^{2} \sum_{i=0}^{\infty} \sum_{j=0}^{\infty}\|\bar{w}\|_{2}^{2}
\end{aligned}
$$

According to Definition 4, we obtain that system (1) is exponentially stable and has weighted $l_{2}$-gain $\gamma$. The proof is completed.

Remark 8 We would like to stress that the $l_{2}$-gain performance analysis problem of $2 \mathrm{D}$ discrete switched systems is firstly considered in the paper. Although some results on $l_{2}$-gain performance analysis of $2 \mathrm{D}$ systems have been obtained in [21-25], the existing methods proposed in these papers cannot be directly applied to $2 \mathrm{D}$ switched systems. In Theorem 2, sufficient conditions for the existence of $l_{2}$-gain performance for system (1) are derived in terms of a set of LMIs.

Remark 9 As for the applicability of Theorem 2, it is easy to see that a larger $\alpha$ and a larger $\gamma$ will be favorable to the feasibility of matrix inequality (19), while a smaller $\alpha$ is more expectable to decrease $\tau_{a}^{*}$, and a smaller $\gamma$ means the better performance of the system. Thus for the first time, we can chose a smaller $\alpha$ and a smaller $\gamma$, and then, by adjusting the values of $\alpha$ and $\gamma$, we can find a feasible solution.

Remark 10 It is noticed that when $\mu=1$ in $\tau_{a}>\tau_{a}^{*}=\frac{\ln \mu}{-\ln \alpha}$, (18) turns out to be $P_{h}^{k}=P_{h}^{l}$, $P_{v}^{k}=P_{v}^{l}, Q_{h}^{k}=Q_{h}^{l}, Q_{v}^{k}=Q_{v}^{l}, W_{h}^{k}=W_{h}^{l}, W_{v}^{k}=W_{v}^{l}, R_{h}^{k}=R_{h}^{l}, R_{v}^{k}=R_{v}^{l}, \forall k, l \in \underline{N}$ and thus a common Lyapunov function exists for all subsystems. Then from (23) we get

$$
\sum_{m=0}^{D-2} \sum_{i+j=m} \alpha^{D-2-i-j} \Gamma(i, j)<0
$$

That is,

$$
\sum_{m=0}^{D-2} \sum_{i+j=m} \alpha^{D-2-i-j}\|\bar{z}\|_{2}^{2}<\gamma^{2} \sum_{m=0}^{D-2} \sum_{i+j=m} \alpha^{D-2-i-j}\|\bar{w}\|_{2}^{2}
$$

Summing $D$ from 2 to $\infty$, we get

$$
\sum_{D=2}^{\infty} \sum_{m=0}^{D-2} \sum_{i+j=m} \alpha^{D-2-i-j}\|\bar{z}\|_{2}^{2}<\gamma^{2} \sum_{D=2}^{\infty} \sum_{m=0}^{D-2} \sum_{i+j=m} \alpha^{D-2-i-j}\|\bar{w}\|_{2}^{2}
$$


Thus

$$
\frac{1}{1-\alpha} \sum_{m=0}^{\infty} \sum_{i+j=m}\|\bar{z}\|_{2}^{2}<\gamma^{2} \frac{1}{1-\alpha} \sum_{m=0}^{\infty} \sum_{i+j=m}\|\bar{w}\|_{2}^{2}
$$

That is,

$$
\sum_{i=0}^{\infty} \sum_{j=0}^{\infty}\|\bar{z}\|_{2}^{2}<\gamma^{2} \sum_{i=0}^{\infty} \sum_{j=0}^{\infty}\|\bar{w}\|_{2}^{2} .
$$

Therefore, $l_{2}$-gain is achieved for switched system (1) under arbitrary switching. We state the fact in the following corollary.

Corollary 1 Consider system (1). For given positive constants $\gamma$ and $\alpha<1$, if there exist positive definite symmetric matrices $P_{h}^{k}=P_{h}, P_{v}^{k}=P_{v}, Q_{h}^{k}=Q_{h}, Q_{v}^{k}=Q_{v}, W_{h}^{k}=W_{h}, W_{v}^{k}=$ $W_{v}, R_{h}^{k}=R_{h}, R_{v}^{k}=R_{v}$, and matrices $N_{1}^{k}=\left[\begin{array}{c}N_{11}^{k} \\ N_{12}^{k}\end{array}\right], N_{2}^{k}=\left[\begin{array}{c}N_{21}^{k} \\ N_{22}^{k}\end{array}\right], M_{1}^{k}=\left[\begin{array}{c}M_{11}^{k} \\ M_{12}^{k}\end{array}\right], M_{2}^{k}=\left[\begin{array}{c}M_{21}^{k} \\ M_{22}^{k}\end{array}\right], X^{k}=$ $\left[\begin{array}{cc}X_{11}^{k} & X_{12}^{k} \\ * & X_{22}^{k}\end{array}\right]>0$ and $Y^{k}=\left[\begin{array}{cc}Y_{11}^{k} & Y_{12}^{k} \\ * & Y_{22}^{k}\end{array}\right]>0$ with appropriate dimensions such that (16) and (19) hold for $k \in \underline{N}$, then the $2 D$ discrete switched system (1) achieves the $l_{2}$-gain under arbitrary switching signals.

\section{Numerical example}

In this section, we present an example to illustrate the effectiveness of the proposed approach. Consider system (1) with parameters as follows:

$$
\begin{aligned}
& A_{1}^{1}=\left[\begin{array}{cc}
0.12 & 0 \\
0 & 0.1
\end{array}\right], \quad A_{2}^{1}=\left[\begin{array}{cc}
0 & 0.01 \\
0 & 0
\end{array}\right], \\
& A_{d 1}^{1}=\left[\begin{array}{cc}
0 & 0.1 \\
0 & 0
\end{array}\right], \quad A_{d 2}^{1}=\left[\begin{array}{ll}
0.025 & 0 \\
0.024 & 0
\end{array}\right], \\
& B_{1}^{1}=\left[\begin{array}{c}
0.012 \\
0.01
\end{array}\right], \quad B_{2}^{1}=\left[\begin{array}{c}
0.014 \\
0
\end{array}\right], \\
& H^{1}=\left[\begin{array}{ll}
0.06 & 0.06
\end{array}\right], \quad L^{1}=0.1, \\
& A_{1}^{2}=\left[\begin{array}{cc}
0 & 0.2 \\
0 & 0.1
\end{array}\right], \quad A_{2}^{2}=\left[\begin{array}{cc}
0 & 0 \\
0.02 & 0
\end{array}\right], \\
& A_{d 1}^{2}=\left[\begin{array}{cc}
0 & 0.022 \\
0 & 0
\end{array}\right], \quad A_{d 2}^{2}=\left[\begin{array}{cc}
0.03 & 0 \\
0 & 0.03
\end{array}\right], \\
& B_{1}^{2}=\left[\begin{array}{c}
0.01 \\
0
\end{array}\right], \quad B_{2}^{2}=\left[\begin{array}{c}
0 \\
0.02
\end{array}\right], \\
& H^{2}=\left[\begin{array}{ll}
0.08 & 0.08
\end{array}\right], \quad L^{2}=0.2, \\
& d_{1}(i)=1+|\sin (0.5 \pi i)|, \quad d_{2}(j)=|\sin (0.5 \pi j)| .
\end{aligned}
$$


According to Remark 9, we can firstly take $\alpha=0.85$ and $\gamma=0.3$, then solving (16) and (19) gives rise to the following solution:

$$
\begin{array}{ll}
P_{h}^{1}=\left[\begin{array}{cc}
16.0149 & -0.0554 \\
-0.0554 & 17.1723
\end{array}\right], & P_{h}^{2}=\left[\begin{array}{cc}
14.7372 & -2.8781 \\
-2.8781 & 15.7325
\end{array}\right], \\
P_{v}^{1}=\left[\begin{array}{cc}
12.4044 & -0.1679 \\
-0.1679 & 12.7975
\end{array}\right], & P_{v}^{2}=\left[\begin{array}{cc}
11.6846 & -1.7028 \\
-1.7028 & 9.9813
\end{array}\right], \\
Q_{h}^{1}=\left[\begin{array}{cc}
1.5578 & -0.0401 \\
-0.0401 & 1.8157
\end{array}\right], & Q_{h}^{2}=\left[\begin{array}{cc}
1.4961 & -0.3441 \\
-0.3441 & 1.1635
\end{array}\right], \\
Q_{v}^{1}=\left[\begin{array}{cc}
1.3303 & -0.0206 \\
-0.0206 & 1.3824
\end{array}\right], & Q_{v}^{2}=\left[\begin{array}{cc}
1.1958 & -0.2237 \\
-0.2237 & 1.0261
\end{array}\right], \\
W_{h}^{1}=\left[\begin{array}{cc}
2.5240 & -0.0129 \\
-0.0129 & 2.6381
\end{array}\right], & W_{h}^{2}=\left[\begin{array}{cc}
2.3174 & -0.2703 \\
-0.2703 & 2.1465
\end{array}\right], \\
W_{v}^{1}=\left[\begin{array}{cc}
2.4935 & -0.0188 \\
-0.0188 & 2.5536
\end{array}\right], & W_{v}^{2}=\left[\begin{array}{cc}
2.3520 & -0.2576 \\
-0.2576 & 2.1315
\end{array}\right], \\
R_{h}^{1}=\left[\begin{array}{cc}
2.3840 & 0.0583 \\
0.0583 & 2.4730
\end{array}\right], & R_{h}^{2}=\left[\begin{array}{cc}
1.8431 & 0.0074 \\
0.0074 & 2.3348
\end{array}\right], \\
R_{v}^{1}=\left[\begin{array}{cc}
2.4817 & -0.0143 \\
-0,0143 & 2.5963
\end{array}\right], & R_{v}^{2}=\left[\begin{array}{cc}
2.4429 & -0.2576 \\
-0.2576 & 2.0604
\end{array}\right],
\end{array}
$$

and $\mu=1.8141$, then we obtain from (17) that $\tau_{a}^{*}=3.65$. Choosing $\tau_{a}=4$, some simulation results are shown in Figures 1-3, where the boundary condition of the system is

$$
x(i, j)=\frac{1}{50(j+1)}, \quad \forall 0 \leq j \leq 20, i=0, \quad x(i, j)=\frac{1}{50(i+1)}, \quad \forall 0 \leq i \leq 20, j=0,
$$

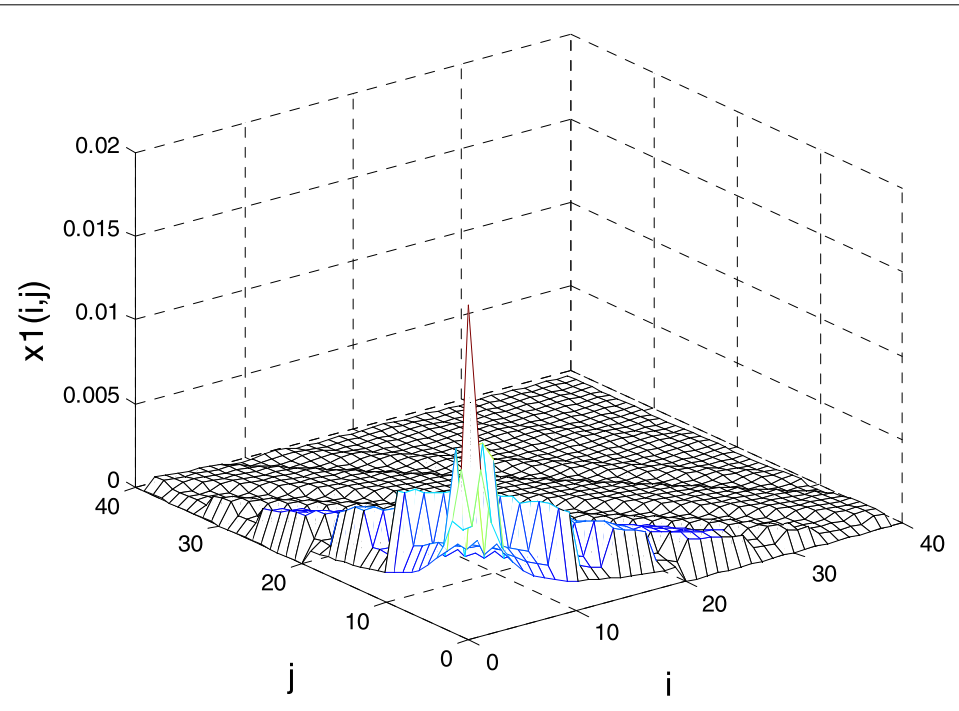

Figure 1 Response of state $x_{1}(i, j)$. 


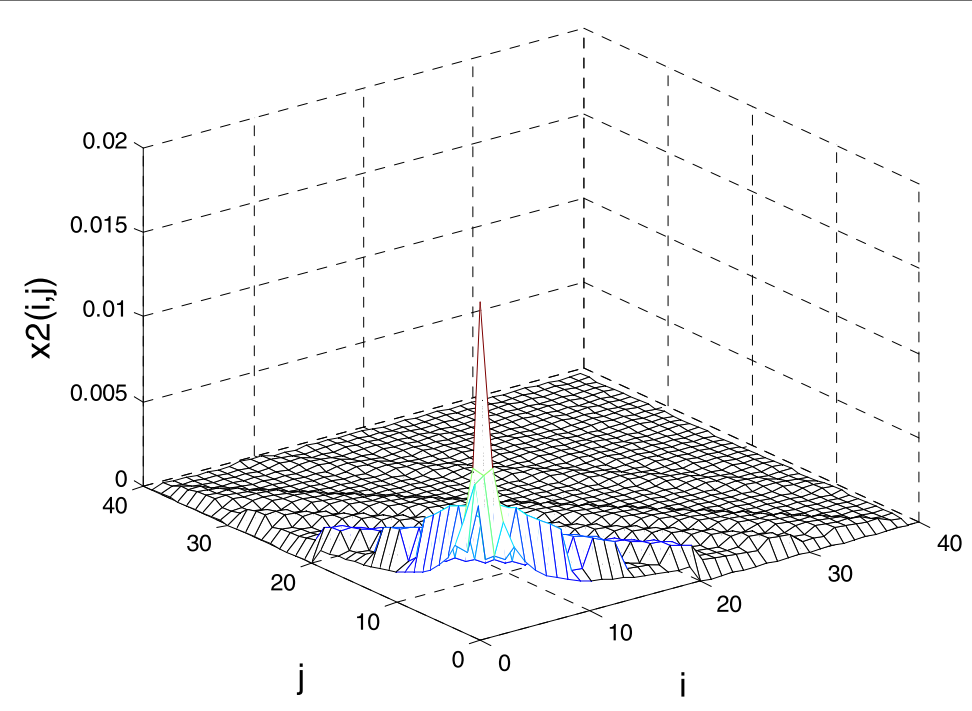

Figure 2 Response of state $x_{2}(i, j)$.

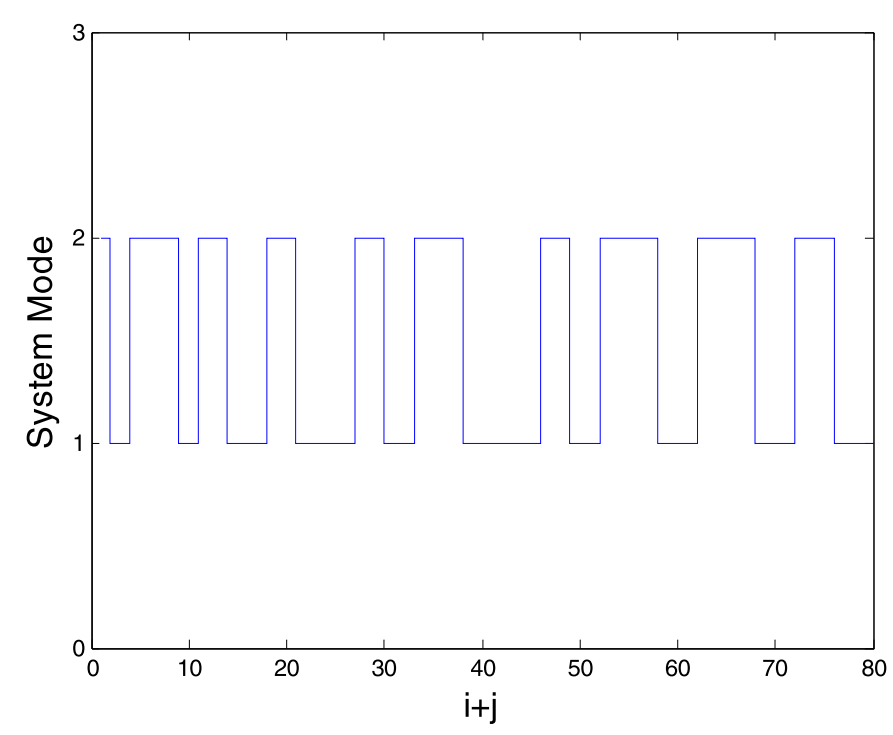

Figure 3 Switching signal.

and $w(i, j)=0.5 \exp (-0.025 \pi(i+j))$. It can be seen from Figures $1-3$ that the system is asymptotically stable. Furthermore, when the boundary condition is zero, by computing, we get $\sum_{i=0}^{\infty} \sum_{j=0}^{\infty} \alpha^{i+j}\|\bar{z}\|_{2}^{2}=0.0867$ and $\sum_{i=0}^{\infty} \sum_{j=0}^{\infty}\|\bar{w}\|_{2}^{2}=14.6952$, and it satisfies condition (2) in Definition 4. Therefore, it can be observed that the system has weighted $l_{2}$-gain $\gamma=0.3$. This demonstrates the effectiveness of the proposed approach.

\section{Conclusions}

This paper has investigated the problems of stability and $l_{2}$-gain analysis for $2 \mathrm{D}$ discrete switched systems with time-varying delays described by the second FM model. A delaydependent exponential stability criterion is obtained via the average dwell time approach. 
Then some sufficient conditions for the existence of weighted $l_{2}$-gain for the considered system are derived in terms of LMIs. Finally, an example is also given to illustrate the applicability of the proposed results. Our future work will focus on extending the proposed results to other kinds of 2D discrete switched systems such as 2D discrete switched stochastic systems or 2D discrete switched nonlinear systems.

\section{Appendix}

Proof of Theorem 1 It is assumed that the $k$ th subsystem is active on the interval $\left[m_{\pi}, m_{\pi+1}\right)$, and the $l$ th subsystem is active on the interval $\left[m_{\pi-1}, m_{\pi}\right)$. Now we consider the Lyapunov function candidate for the $k$ th subsystem

$$
V_{k}(x(i, j))=V_{k}^{h}(x(i, j))+V_{k}^{v}(x(i, j)),
$$

where

$$
\begin{aligned}
& V_{k}^{h}(x(i, j))=\sum_{g=1}^{5} V_{g k}^{h}(x(i, j)), \\
& V_{1 k}^{h}(i, j)=x(i, j)^{T} P_{h}^{k} x(i, j), \\
& V_{2 k}^{h}(x(i, j))=\sum_{r=i-d_{1}(i)}^{i-1} x(r, j)^{T} Q_{h}^{k} x(r, j) \alpha^{i-r-1}, \\
& V_{3 k}^{h}(x(i, j))=\sum_{r=i-\bar{d}_{1}}^{i-1} x(r, j)^{T} W_{h}^{k} x(r, j) \alpha^{i-r-1}, \\
& V_{4 k}^{h}(x(i, j))=\sum_{s=-\bar{d}_{1}+1}^{-\underline{d}_{1}} \sum_{r=i+s}^{i-1} x(r, j)^{T} Q_{h}^{k} x(r, j) \alpha^{i-r-1}, \\
& V_{5 k}^{h}(x(i, j))=\sum_{s=-\bar{d}_{1}+1}^{0} \sum_{r=i+s-1}^{i-1} \eta(r, j)^{T} R_{h}^{k} \eta(r, j) \alpha^{i-r-1}, \\
& V_{k}^{v}(x(i, j))=\sum_{g=1}^{5} V_{g k}^{v}(x(i, j)), \\
& V_{1 k}^{v}(x(i, j))=x(i, j)^{T} P_{v}^{k} x(i, j), \\
& V_{3 k}^{v}(x(i, j))=\sum_{t=j-d_{2}(j)}^{j-1} x(i, t)^{T} Q_{v}^{k} x(i, t) \alpha^{j-t-1}, \\
& V_{3 k}^{v}(x(i, j))=\sum_{t=j-\bar{d}_{2}}^{j-1} x(i, t)^{T} W_{v}^{k} x(i, t) \alpha^{j-t-1}, \\
& V_{4 k}^{v}(x(i, j))=\sum_{s=-\bar{d}_{2}+1}^{-\underline{d}_{2}} \sum_{t=j+s}^{j-1} x(i, t)^{T} Q_{\nu}^{k} x(i, t) \alpha^{j-t-1}, \\
& V_{5 k}^{v}(x(i, j))=\sum_{s=-\bar{d}_{2}+1}^{0} \sum_{t=j+s-1}^{j-1} \delta(i, t)^{T} R_{v}^{k} \delta(i, t) \alpha^{j-t-1},
\end{aligned}
$$




$$
\begin{aligned}
& \eta(r, j)=x(r+1, j)-x(r, j), \\
& \delta(i, t)=x(i, t+1)-x(i, t) .
\end{aligned}
$$

Then we have

$$
\begin{aligned}
& V_{1 k}^{h}(i+1, j+1)-\alpha V_{1 k}^{h}(i, j+1) \\
& =x^{T}(i+1, j+1) P_{h}^{k} x(i+1, j+1)-\alpha x^{T}(i, j+1) P_{h}^{k} x(i, j+1), \\
& V_{2 k}^{h}(i+1, j+1)-\alpha V_{2 k}^{h}(i, j+1) \\
& \leq x(i, j+1)^{T} Q_{h}^{k} x(i, j+1)-x\left(i-d_{1}(i), j+1\right)^{T} Q_{h}^{k} x\left(i-d_{1}(i), j+1\right) \alpha^{\bar{d}_{1}} \\
& +\sum_{r=i+1-\bar{d}_{1}}^{i-\underline{d}_{1}} x(r, j+1)^{T} Q_{h}^{k} x(r, j+1) \alpha^{i-r}, \\
& V_{3 k}^{h}(i+1, j+1)-\alpha V_{3 k}^{h}(i, j+1) \\
& =x(i, j+1)^{T} W_{h}^{k} x(i, j+1)-x\left(i-\bar{d}_{1}, j+1\right)^{T} W_{h}^{k} x\left(i-\bar{d}_{1}, j+1\right) \alpha^{\bar{d}_{1},} \\
& V_{4 k}^{h}(i+1, j+1)-\alpha V_{4 k}^{h}(i, j+1) \\
& =\left(\bar{d}_{1}-\underline{d}_{1}\right) x(i, j+1)^{T} Q_{h}^{k} x(i, j+1)-\sum_{r=i-\bar{d}_{1}+1}^{i-\underline{d}_{1}} x(r, j+1)^{T} Q_{h}^{k} x(r, j+1) \alpha^{i-r}, \\
& V_{5 k}^{h}(i+1, j+1)-\alpha V_{5 k}^{h}(i, j+1) \\
& \leq \bar{d}_{1} \eta(i, j+1)^{T} R_{h}^{k} \eta(i, j+1)-\sum_{r=i-\bar{d}_{1}}^{i-1} \eta(r, j+1)^{T} R_{h}^{k} \eta(r, j+1) \alpha^{\bar{d}_{1}}, \\
& V_{1 k}^{v}(i+1, j+1)-\alpha V_{1 k}^{v}(i+1, j) \\
& =x^{T}(i+1, j+1) P_{v}^{k} x(i+1, j+1)-\alpha x^{T}(i+1, j) P_{v}^{k} x(i+1, j), \\
& V_{2 k}^{v}(i+1, j+1)-\alpha V_{2 k}^{v}(i+1, j) \\
& \leq x(i+1, j)^{T} Q_{\nu}^{k} x(i+1, j)-x\left(i+1, j-d_{2}(j)\right)^{T} Q_{\nu}^{k} x\left(i+1, j-d_{2}(j)\right) \alpha^{\bar{d}_{2}} \\
& +\sum_{t=j+1-\bar{d}_{2}}^{j-\underline{d}_{2}} x(i+1, t)^{T} Q_{\nu}^{k} x(i+1, t) \alpha^{j-t}, \\
& V_{3 k}^{v}(i+1, j+1)-\alpha V_{3 k}^{v}(i+1, j) \\
& =x(i+1, j)^{T} W_{v}^{k} x(i+1, j)-x\left(i+1, j-\bar{d}_{2}\right)^{T} W_{v}^{k} x\left(i+1, j-\bar{d}_{2}\right) \alpha^{\bar{d}_{2}}, \\
& V_{4 k}^{v}(i+1, j+1)-\alpha V_{4 k}^{v}(i+1, j) \\
& =\left(\bar{d}_{2}-\underline{d}_{2}\right) x(i+1, j)^{T} Q_{\nu}^{k} x(i+1, j)-\sum_{t=j+1-\bar{d}_{2}}^{j-\underline{d}_{2}} x(i+1, t)^{T} Q_{\nu}^{k} x(i+1, t) \alpha^{j-t}, \\
& V_{5 k}^{v}(i+1, j+1)-\alpha V_{5 k}^{v}(i+1, j) \\
& \leq \bar{d}_{2} \delta(i+1, j)^{T} R_{v}^{k} \delta(i+1, j)-\sum_{t=j-\bar{d}_{2}}^{j-1} \delta(i+1, t)^{T} R_{v}^{k} \delta(i+1, t) \alpha^{\bar{d}_{2}}
\end{aligned}
$$




$$
\begin{aligned}
\eta(i, j+1)= & x(i+1, j+1)-x(i, j+1) \\
= & \left(A_{1}^{k}-I\right) x(i, j+1)+A_{2}^{k} x(i+1, j)+A_{d 1}^{k} x\left(i-d_{1}(i), j+1\right) \\
& +A_{d 2}^{k} x\left(i+1, j-d_{2}(j)\right)+B_{1}^{k} w(i, j+1)+B_{2}^{k} w(i+1, j), \\
\delta(i+1, j)= & x(i+1, j+1)-x(i+1, j) \\
= & A_{1}^{k} x(i, j+1)+\left(A_{2}^{k}-I\right) x(i+1, j)+A_{d 1}^{k} x\left(i-d_{1}(i), j+1\right) \\
& +A_{d 2}^{k} x\left(i+1, j-d_{2}(j)\right)+B_{1}^{k} w(i, j+1)+B_{2}^{k} w(i+1, j) .
\end{aligned}
$$

For simplicity, we denote

$$
\begin{aligned}
& V_{k}^{h}(i, j)=V_{k}^{h}(x(i, j)), \quad V_{k}^{v}(i, j)=V_{k}^{v}(x(i, j)), \quad V_{k}(i, j)=V_{k}(x(i, j)), \\
& V_{k}^{h}(i, j+1)=V_{k}^{h}(x(i, j+1)), \quad V_{k}^{v}(i+1, j)=V_{k}^{v}(x(i+1, j)), \\
& V_{k}^{h}(i+1, j+1)=V_{k}^{h}(x(i+1, j+1)), \quad V_{k}^{v}(i+1, j+1)=V_{k}^{v}(x(i+1, j+1)) .
\end{aligned}
$$

Now let us discuss the case where $w(i, j)=0$. It follows from (28) that

$$
\begin{aligned}
& V_{k}^{h}(i+1, j+1)+V_{k}^{v}(i+1, j+1)-\alpha\left(V_{k}^{h}(i, j+1)+V_{k}^{v}(i+1, j)\right) \\
& \leq\left[\begin{array}{c}
x(i, j+1) \\
x(i+1, j) \\
x\left(i-d_{1}(i), j+1\right) \\
x\left(i+1, j-d_{2}(j)\right) \\
x\left(i-\bar{d}_{1}, j+1\right) \\
x\left(i+1, j-\bar{d}_{2}\right)
\end{array}\right]^{T}\left(\Psi+\left[\begin{array}{c}
A_{1}^{k T} \\
A_{2}^{k T} \\
A_{d 1}^{k T} \\
A_{d 2}^{k T} \\
0 \\
0
\end{array}\right]\left(P_{h}^{k}+P_{v}^{k}\right)\left[\begin{array}{c}
A_{1}^{k T} \\
A_{2}^{k T} \\
A_{d 1}^{k T} \\
A_{d 2}^{k T} \\
0 \\
0
\end{array}\right]\right)\left[\begin{array}{c}
x(i, j+1) \\
x(i+1, j) \\
x\left(i-d_{1}(i), j+1\right) \\
x\left(i+1, j-d_{2}(j)\right) \\
x\left(i-\bar{d}_{1}, j+1\right) \\
x\left(i+1, j-\bar{d}_{2}\right)
\end{array}\right] \\
& +\bar{d}_{1} \eta(i, j+1)^{T} R_{h}^{k} \eta(i, j+1)-\sum_{r=i-\bar{d}_{1}}^{i-1} \eta(r, j+1)^{T} R_{h}^{k} \eta(r, j+1) \alpha^{\bar{d}_{1}} \\
& +\bar{d}_{2} \delta(i+1, j)^{T} R_{v}^{k} \delta(i+1, j)-\sum_{t=j-\bar{d}_{2}}^{j-1} \delta(i+1, t)^{T} R_{v}^{k} \delta(i+1, t) \alpha^{\bar{d}_{2},},
\end{aligned}
$$

where

$$
\begin{aligned}
& \Psi=\operatorname{diag}\left\{\Psi_{11}, \Psi_{22},-\alpha^{\bar{d}_{1}} Q_{h}^{k},-\alpha^{\bar{d}_{2}} Q_{v}^{k},-\alpha^{\bar{d}_{1}} W_{h}^{k},-\alpha^{\bar{d}_{2}} W_{v}^{k}\right\}, \\
& \Psi_{11}=-\alpha P_{h}^{k}+W_{h}^{k}+\left(\bar{d}_{1}-\underline{d}_{1}+1\right) Q_{h}^{k}, \quad \Psi_{22}=-\alpha P_{v}^{k}+W_{v}^{k}+\left(\bar{d}_{2}-\underline{d}_{2}+1\right) Q_{v}^{k} .
\end{aligned}
$$

Notice that the following equations hold for any matrices $N_{1}^{k}=\left[\begin{array}{c}N_{11}^{k} \\ N_{12}^{k}\end{array}\right], N_{2}^{k}=\left[\begin{array}{c}N_{21}^{k} \\ N_{22}^{k}\end{array}\right], M_{1}^{k}=$ $\left[\begin{array}{c}M_{11}^{k} \\ M_{12}^{k}\end{array}\right]$, and $M_{2}^{k}=\left[\begin{array}{c}M_{21}^{k} \\ M_{22}^{k}\end{array}\right]$ with appropriate dimensions:

$$
\begin{aligned}
0= & 2 \alpha^{\bar{d}_{1}}\left[x^{T}(i, j+1) N_{11}^{k}+x^{T}\left(i-d_{1}(i), j+1\right) N_{12}^{k}\right] \\
& \times\left[x(i, j+1)-x\left(i-d_{1}(i), j+1\right)-\sum_{r=i-d_{1}(i)}^{i-1} \eta(r, j+1)\right],
\end{aligned}
$$




$$
\begin{aligned}
0= & 2 \alpha^{\bar{d}_{2}}\left[x^{T}(i+1, j) M_{21}^{k}+x^{T}\left(i+1, j-d_{2}(j)\right) M_{22}^{k}\right] \\
& \times\left[x\left(i+1, j-d_{2}(j)\right)-x\left(i+1, j-\bar{d}_{2}\right)-\sum_{t=j-\bar{d}_{2}}^{j-d_{2}(j)-1} \delta(i+1, t)\right] .
\end{aligned}
$$

On the other hand, for any matrices $X^{k}=\left[\begin{array}{cc}X_{11}^{k} & X_{12}^{k} \\ * & X_{22}^{k}\end{array}\right]>0$ and $Y^{k}=\left[\begin{array}{cc}Y_{11}^{k} & Y_{12}^{k} \\ * & Y_{22}^{k}\end{array}\right]>0$, the following equations also hold:

$$
\begin{aligned}
0= & \bar{d}_{1} \alpha^{\bar{d}_{1}}\left[\begin{array}{c}
x(i, j+1) \\
x\left(i-d_{1}(i), j+1\right)
\end{array}\right]^{T} X^{k}\left[\begin{array}{c}
x(i, j+1) \\
x\left(i-d_{1}(i), j+1\right)
\end{array}\right] \\
& -\sum_{r=i-d_{1}(i)}^{i-1}\left[\begin{array}{c}
x(i, j+1) \\
x\left(i-d_{1}(i), j+1\right)
\end{array}\right]^{T} X^{k}\left[\begin{array}{c}
x(i, j+1) \\
x\left(i-d_{1}(i), j+1\right)
\end{array}\right] \alpha^{\bar{d}_{1}} \\
& -\sum_{r=i-\bar{d}_{1}}^{i-1-d_{1}(i)}\left[\begin{array}{c}
x(i, j+1) \\
x\left(i-d_{1}(i), j+1\right)
\end{array}\right]^{T} X^{k}\left[\begin{array}{c}
x(i, j+1) \\
x\left(i-d_{1}(i), j+1\right)
\end{array}\right] \alpha^{\bar{d}_{1},} \\
0= & \bar{d}_{2} \alpha^{\bar{d}_{2}}\left[\begin{array}{c}
x(i+1, j) \\
x\left(i+1, j-d_{2}(j)\right)
\end{array}\right]^{T} Y^{k}\left[\begin{array}{c}
x(i+1, j) \\
x\left(i+1, j-d_{2}(j)\right)
\end{array}\right] \\
& -\sum_{t=j-d_{2}(j)}^{j-1}\left[\begin{array}{c}
x(i+1, j) \\
x\left(i+1, j-d_{2}(j)\right)
\end{array}\right]^{T} Y^{k}\left[\begin{array}{c}
x(i+1, j) \\
x\left(i+1, j-d_{2}(j)\right)
\end{array}\right] \alpha^{\bar{d}_{2}} \\
& -\sum_{t=j-\bar{d}_{2}}^{j-d_{2}(j)}\left[\begin{array}{c}
x(i+1, j) \\
x\left(i+1, j-d_{2}(j)\right)
\end{array}\right]^{T} Y^{k}\left[\begin{array}{c}
x(i+1, j) \\
x\left(i+1, j-d_{2}(j)\right)
\end{array}\right] \alpha^{\bar{d}_{2} .}
\end{aligned}
$$

Adding the terms on the right-hand sides of equations (30)-(35) to (29), allows us to write (29) as

$$
\begin{gathered}
V_{k}^{h}(i+1, j+1)+V_{k}^{v}(i+1, j+1)-\alpha\left(V_{k}^{h}(i, j+1)+V_{k}^{v}(i+1, j)\right) \\
\leq\left[\begin{array}{c}
x(i, j+1) \\
x(i+1, j) \\
x\left(i-d_{1}(i), j+1\right) \\
x\left(i+1, j-d_{2}(j)\right) \\
x\left(i-\bar{d}_{1}, j+1\right) \\
x\left(i+1, j-\bar{d}_{2}\right)
\end{array}\right]^{T}\left(\Psi+\left[\begin{array}{c}
A_{1}^{k T} \\
A_{2}^{k T} \\
A_{d 1}^{k T} \\
A_{d 2}^{k T} \\
0 \\
0
\end{array}\right]\left(P_{h}^{k}+P_{v}^{k}\right)\left[\begin{array}{c}
A_{1}^{k T} \\
A_{2}^{k T} \\
A_{d 1}^{k T} \\
A_{d 2}^{k T} \\
0 \\
0
\end{array}\right]^{T}\right.
\end{gathered}
$$




$$
\begin{aligned}
& \left.+\left[\begin{array}{cc}
A_{1}^{k T}-I & A_{1}^{k T} \\
A_{2}^{k T} & A_{2}^{k T}-I \\
A_{d 1}^{k T} & A_{d 1}^{k T} \\
A_{d 2}^{k T} & A_{d 2}^{k T} \\
0 & 0 \\
0 & 0
\end{array}\right]\left[\begin{array}{cc}
\bar{d}_{1} R_{h}^{k} & 0 \\
0 & \bar{d}_{2} R_{v}^{k}
\end{array}\right]\left[\begin{array}{cc}
A_{1}^{k T}-I & A_{1}^{k T} \\
A_{2}^{k T} & A_{2}^{k T}-I \\
A_{d 1}^{k T} & A_{d 1}^{k T} \\
A_{d 2}^{k T} & A_{d 2}^{k T} \\
0 & 0 \\
0 & 0
\end{array}\right]\right)^{T} \\
& \times\left[\begin{array}{c}
x(i, j+1) \\
x(i+1, j) \\
x\left(i-d_{1}(i), j+1\right) \\
x\left(i+1, j-d_{2}(j)\right) \\
x\left(i-\bar{d}_{1}, j+1\right) \\
x\left(i+1, j-\bar{d}_{2}\right)
\end{array}\right] \\
& -\sum_{r=i-d_{1}(i)}^{i-1}\left[\begin{array}{c}
x(i, j+1) \\
\left(i-d_{1}(i), j+1\right) \\
\eta(r, j+1)
\end{array}\right]^{T}\left[\begin{array}{cc}
X^{k} & N_{1}^{k} \\
* & R_{h}^{k}
\end{array}\right]\left[\begin{array}{c}
x(i, j+1) \\
\left(i-d_{1}(i), j+1\right) \\
\eta(r, j+1)
\end{array}\right] \alpha^{\bar{d}_{1}} \\
& -\sum_{r=i-\bar{d}_{1}}^{i-d_{1}(i)-1}\left[\begin{array}{c}
x(i, j+1) \\
x\left(i-d_{1}(i), j+1\right) \\
\eta(r, j+1)
\end{array}\right]^{T}\left[\begin{array}{cc}
X^{k} & N_{2}^{k} \\
* & R_{h}^{k}
\end{array}\right]\left[\begin{array}{c}
x(i, j+1) \\
x\left(i-d_{1}(i), j+1\right) \\
\eta(r, j+1)
\end{array}\right] \alpha^{\bar{d}_{1}} \\
& -\sum_{t=j-d_{2}(j)}^{j-1}\left[\begin{array}{c}
x(i+1, j) \\
x\left(i+1, j-d_{2}(j)\right) \\
\delta(i+1, t)
\end{array}\right]^{T}\left[\begin{array}{cc}
Y^{k} & M_{1}^{k} \\
* & R_{v}^{k}
\end{array}\right]\left[\begin{array}{c}
x(i+1, j) \\
x\left(i+1, j-d_{2}(j)\right) \\
\delta(i+1, t)
\end{array}\right] \alpha^{\bar{d}_{2}} \\
& -\sum_{t=j-\bar{d}_{2}}^{j-d_{2}(j)-1}\left[\begin{array}{c}
x(i+1, j) \\
x\left(i+1, j-d_{2}(j)\right) \\
\delta(i+1, t)
\end{array}\right]^{T}\left[\begin{array}{cc}
Y^{k} & M_{2}^{k} \\
* & R_{v}^{k}
\end{array}\right]\left[\begin{array}{c}
x(i+1, j) \\
x\left(i+1, j-d_{2}(j)\right) \\
\delta(i+1, t)
\end{array}\right] \alpha^{\bar{d}_{2}} .
\end{aligned}
$$

Thus it follows from (15)-(16) that

$$
V_{k}^{h}(i+1, j+1)+V_{k}^{v}(i+1, j+1)<\alpha\left(V_{k}^{h}(i, j+1)+V_{k}^{v}(i+1, j)\right) .
$$

When $D \geq m_{\pi}>z=\max \left(z_{1}, z_{2}\right)$, we have $V_{k}^{h}(0, D)=V_{k}^{v}(D, 0)=0$. Then summing up both sides of (37) from $D-2$ to 0 with respect to $j$ and 0 to $D-2$ with respect to $i$, one gets

$$
\begin{aligned}
\sum_{i+j=D} V_{k}(i, j)= & V_{k}^{h}(0, D)+V_{k}^{h}(1, D-1)+V_{k}^{h}(2, D-2)+\cdots+V_{k}^{h}(D-1,1)+V_{k}^{h}(D, 0) \\
& +V_{k}^{v}(0, D)+V_{k}^{v}(1, D-1)+V_{k}^{v}(2, D-2) \\
& +\cdots+V_{k}^{v}(D-1,1)+V_{k}^{v}(D, 0) \\
< & \alpha\left(V_{k}^{h}(0, D-1)+V_{k}^{v}(0, D-1)+V_{k}^{h}(1, D-2)+V_{k}^{v}(1, D-2)\right. \\
& \left.+\cdots+V_{k}^{h}(D-1,0)+V_{k}^{v}(D-1,0)\right) \\
= & \alpha \sum_{i+j=D-1} V_{k}(i, j) .
\end{aligned}
$$


Thus (8) can be directly obtained. Moreover, by (28), we can find two positive scalars $\lambda_{1}$ and $\lambda_{2}$ such that (7) holds, where

$$
\begin{aligned}
\lambda_{1}= & \min _{k \in \underline{N}}\left(\lambda_{\min }\left(P_{h}^{k}\right)+\lambda_{\min }\left(P_{v}^{k}\right)\right), \\
\lambda_{2}= & \max _{k \in \underline{N}}\left(\lambda_{\max }\left(P_{h}^{k}\right)+\lambda_{\max }\left(P_{v}^{k}\right)+\bar{d}_{1} \lambda_{\max }\left(Q_{h}^{k}\right)+\bar{d}_{2} \lambda_{\max }\left(Q_{v}^{k}\right)+\bar{d}_{1} \lambda_{\max }\left(W_{h}^{k}\right)\right. \\
& +\bar{d}_{2} \lambda_{\max }\left(W_{v}^{k}\right)+\left(\bar{d}_{1}-\underline{d}_{1}\right)^{2} \lambda_{\max }\left(Q_{h}^{k}\right) \\
& \left.+\left(\bar{d}_{2}-\underline{d}_{2}\right)^{2} \lambda_{\max }\left(Q_{v}^{k}\right)+\bar{d}_{1}^{2} \lambda_{\max }\left(R_{h}^{k}\right)+\bar{d}_{2}^{2} \lambda_{\max }\left(R_{v}^{k}\right)\right) .
\end{aligned}
$$

In addition, (9) can be deduced from (18), thus by Lemma 1, we can conclude that 2D discrete switched system (1) is exponentially stable.

\section{Competing interests}

The authors declare that they have no competing interests.

\section{Authors' contributions}

SH carried out the main results of this article and drafted the manuscript. ZX directed the study and helped with the inspection. All the authors read and approved the final manuscript.

\section{Acknowledgements}

This research was supported by the National Natural Science Foundation of China under Grant Nos. 60974027 and 61273120

Received: 3 November 2012 Accepted: 22 February 2013 Published: 14 March 2013

\section{References}

1. Du, CL, Xie, LH: Control and Filtering of Two-Dimensional Systems. Springer, Berlin (2002)

2. Kaczorek, T: Two-Dimensional Linear Systems. Springer, Berlin (1985)

3. Lu, WS: Two-Dimensional Digital Filters. Dekker, New York (1992)

4. Owens, DH, Amann, N, Rogers, E, French, M: Analysis of linear iterative learning control schemes - a 2D systems/repetitive processes approach. Multidimens. Syst. Signal Process. 11(1-2), 125-177 (2000)

5. Li, XD, Ho, JKL, Chow, TWS: Iterative learning control for linear time-variant discrete systems based on 2-D system theory. IEE Proc., Control Theory Appl. 152(1), 13-18 (2005)

6. Bochniak, J, Galkowski, K, Rogers, E, Mehdi, D, Kummert, O, Bachelier, A: Stabilization of discrete linear repetitive processes with switched dynamics. Multidimens. Syst. Signal Process. 17(2-3), 271-295 (2006)

7. Galkowski, K, Rogers, E, Xu, S, Lam, J, Owens, DH: LMls - a fundamental tool in analysis and controller design for discrete linear repetitive processes. IEEE Trans. Circuits Syst. I, Fundam. Theory Appl. 49(6), 768-777 (2002)

8. $\mathrm{Xu}, \mathrm{L}, \mathrm{Wu}, \mathrm{LK}, \mathrm{Wu}, \mathrm{QH}$, Lin, ZP, Xiao, YG: On realization of 2D discrete systems by Fornasini-Marchesini model. Int. J. Control. Autom. Syst. 3(4), 631-639 (2005)

9. Xu, L, Wu, QH, Lin, ZP, Xiao, YG: A new constructive procedure for 2-D coprime realization in Fornasini-Marchesini model. IEEE Trans. Circuits Syst. 54(9), 2061-2069 (2007)

10. Lin, ZP: Feedback stabilization of MIMO nD linear systems. IEEE Trans. Autom. Control 45(12), $2419-2424$ (2000)

11. Xu, L, Yamada, M, Lin, ZP, Saito, O, Anazawa, Y: Further improvements on Bose's 2D stability test. Int. J. Control. Autom. Syst. 2(3), 319-332 (2004)

12. Lin, ZP, Lam, J, Galkowski, K, Xu, SY: A constructive approach to stabilizability and stabilization of a class of $n D$ systems. Multidimens. Syst. Signal Process. 12(3-4), 329-343 (2001)

13. Fornasini, E, Marchesini, G: Stability analysis of 2-D systems. IEEE Trans. Circuits Syst. 27(10), 1210-1217 (1980)

14. Ye, SX, Wang, WQ: Stability analysis and stabilisation for a class of 2-D nonlinear discrete systems. Int. J. Syst. Sci. 42(5), 839-851 (2011)

15. Paszke, W, Lam, J, Galkowski, K, Xu, SY, Lin, ZP: Robust stability and stabilisation of 2D discrete state-delayed systems. Syst. Control Lett. 51(3-4), 277-291 (2004)

16. Chen, SF: Delay-dependent stability for 2D systems with time-varying delay subject to state saturation in the Roesser model. Appl. Math. Comput. 216(9), 2613-2622 (2010)

17. Feng, $Z Y, X u, L, W u, M, H e, Y$ : Delay-dependent robust stability and stabilisation of uncertain two-dimensional discrete systems with time-varying delays. IET Control Theory Appl. 4(10), 1959-1971 (2010)

18. $\mathrm{Xu}, \mathrm{HL}$, Lin, ZP, Makur, A: The existence and design of functional observers for two-dimensional systems. Syst. Control Lett. 61(2), 362-368 (2012)

19. Xu, SY, Lam, J, Zhou, Y, Lin, ZP, Paszke, W: Robust $H_{\infty}$ filtering for uncertain 2-D continuous systems. IEEE Trans. Signal Process. 35(5), 1731-1738 (2005)

20. $\mathrm{Xu}, \mathrm{HL}$, Lin, ZP, Makur, A: Non-fragile $H_{\infty}$ filter design for polytopic 2-D system in Fornasini-Marchesini model. In: Proceedings of the 2010 IEEE International Symposium on Circuist and Systems, pp. 997-1000 (2010) 
21. Xie, LH, Du, CL, Soh, YC, Zhang, CS: $H_{\infty}$ and robust control of 2-D systems in FM second model. Multidimens. Syst. Signal Process. 13(3), 265-287 (2002)

22. Du, CL, Xie, LH, Zhang, CS: $H_{\infty}$ control and robust stabilization of two-dimensional systems in Roesser models. Automatica 37(2), 205-211 (2001)

23. Yang, $\mathrm{R}$, Xie, $\mathrm{LH}$, Zhang, $\mathrm{CS}: \mathrm{H}_{2}$ and mixed $\mathrm{H}_{2} / \mathrm{H}_{\infty}$ control of two-dimensional systems in Roesser model. Automatica 42(9), 1507-1514 (2006)

24. Xu, JM, Yu, L: Ho control of 2-D discrete state delay systems. Int. J. Control. Autom. Syst. 4(4), 516-523 (2006)

25. $\mathrm{Xu}, \mathrm{JM}, \mathrm{Yu}, \mathrm{L}$ : Delay-dependent $H_{\infty}$ control for 2-D discrete state delay systems in the second FM model. Multidimens. Syst. Signal Process. 20(4), 333-349 (2009)

26. Hespanha, JP, Morse, AS: Stability of switched systems with average dwell time. In: Proceedings of the 38th IEEE Conference on Decision and Control, pp. 2655-2660 (1999)

27. Zhai, GS, Hu, B, Yasuda, K, Michel, AN: Stability analysis of switched systems with stable and unstable subsystems: an average dwell time approach. In: Proceedings of the American Control Conference, pp. 200-204 (2000)

28. Xiang, ZR, Wang, RH: Robust stabilization of switched non-linear systems with time-varying delays under asynchronous switching. Proc. Inst. Mech. Eng., Part I, J. Syst. Control Eng. 223(8), 1111-1128 (2009)

29. Xiang, ZR, Wang, RH: Robust control for uncertain switched non-linear systems with time delay under asynchronous switching. IET Control Theory Appl. 3(8), 1041-1050 (2009)

30. Sun, XM, Zhao, J, David, JH: Stability and $L_{2}$-gain analysis for switched delay systems: a delay-dependent method. Automatica 42(10), 1769-1774 (2006)

31. Wang, YJ, Yao, ZX, Zuo, ZQ, Zhao, HM: Delay-dependent robust $H_{\infty}$ control for a class of switched systems with time delay. In: IEEE International Symposium on Intelligent Control, pp. 882-887 (2008)

32. Sun, YG, Wang, L, Xie, GM: Delay-dependent robust stability and $H_{\infty}$ control for uncertain discrete-time switched systems with mode-dependent time delays. Appl. Math. Comput. 187(2), 1228-1237 (2007)

33. Wang, $\mathrm{R}$, Liu, $\mathrm{M}, \mathrm{Zhao}, \mathrm{J}$ : Reliable $\mathrm{H}_{\infty}$ control for a class of switched nonlinear systems with actuator failures. Nonlinear Anal. Hybrid Syst. 1(3), 317-325 (2007)

34. Benzaouia, A, Hmamed, A, Tadeo, F: Stability conditions for discrete 2D switching systems, based on a multiple Lyapunov function. In: European Control Conference, pp. 23-26 (2009)

35. Benzaouia, A, Hmamed, A, Tadeo, F, Hajjaji, AE: Stabilisation of discrete 2D time switching systems by state feedback control. Int. J. Syst. Sci. 42(3), 479-487 (2011)

36. Xiang, Z, Huang, S: Stability analysis and stabilization of discrete-time 2D switched systems. Circuits Syst. Signal Process. 32(1), 401-414 (2013)

doi:10.1186/1687-1847-2013-56

Cite this article as: Huang and Xiang: Stability and $/ 2$-gain analysis for 2D discrete switched systems with

time-varying delays in the second FM model. Advances in Difference Equations 2013 2013:56.

\section{Submit your manuscript to a SpringerOpen ${ }^{\odot}$ journal and benefit from:}

- Convenient online submission

- Rigorous peer review

- Immediate publication on acceptance

- Open access: articles freely available online

- High visibility within the field

- Retaining the copyright to your article 